\title{
MULTIPLIER IDEALS AND MODULES ON TORIC VARIETIES
}

\author{
MANUEL BLICKLE
}

\begin{abstract}
A formula computing the multiplier ideal of a monomial ideal on an arbitrary affine toric variety is given. Variants for the multiplier module and test ideals are also treated.
\end{abstract}

\section{INTRODUCTION AND RESULTS}

In this note I generalize Howald's formula How01 for the multiplier ideal of monomial ideals in a polynomial ring to the case of torus invariant ideals in an arbitrary (normal) toric variety. If $\mathfrak{a} \subseteq k\left[x_{1}, \ldots, x_{n}\right]$ is a monomial ideal, his formula computes the multiplier ideal:

$$
\left.\mathcal{J}\left(\mathfrak{a}^{c}\right)=\left\langle x^{v}\right| v+(1, \ldots, 1) \in \text { interior of } c \operatorname{Newt}(\mathfrak{a})\right\rangle
$$

Here and henceforth, $x^{v}$ is shorthand for $x_{1}^{v_{1}} \cdot \ldots \cdot x_{n}^{v_{n}}$ which is a monomial in $k\left[x_{1}, \ldots, x_{n}\right]$. To each monomial $x^{v}$ one associates its exponent vector $v=\left(v_{1}, \ldots, v_{n}\right)$ inside the lattice of exponents $M \cong \mathbb{Z}^{n}$. In this way we assign to a monomial ideal $\mathfrak{a}$ the set of exponents of monomials in $\mathfrak{a}$; the convex hull of this set in $M_{\mathbb{R}}=M \otimes_{\mathbb{Z}} \mathbb{R}$ is the Newton Polyhedron, Newt(a), of $\mathfrak{a}$.

1.1. Multiplier ideal on a pair. The generalization of this formula involves the multiplier ideal of a singular, not necessarily $\mathbb{Q}$-Gorenstein, variety. Let us recall the setup, for details consult Laz, Chapter 9. Let $(X, \Delta)$ be a pair, consisting of a normal variety $X$ and a $\mathbb{Q}$-divisor $\Delta$ such that $K_{X}+\Delta$ is $\mathbb{Q}$-Cartier. This is the situation in which multiplier ideals can be defined.

Definition 1. For such pair $(X, \Delta)$ and an ideal sheaf $\mathfrak{a}$ on $X$, choose a log resolution $\mu: Y \rightarrow X$ of $\mathfrak{a}$ which at the same time is a log resolution of the pair. In particular, $\mathfrak{a} \cdot \mathcal{O}_{Y}=\mathcal{O}_{Y}(-A)$ for some effective normal crossing divisor $A$. Then for all $c>0$, the multiplier ideal of $\mathfrak{a}^{c}$ on the pair $(X, \Delta)$ is

$$
\mathcal{J}\left((X, \Delta), \mathfrak{a}^{c}\right)=\mu_{*} \mathcal{O}_{Y}\left(K_{Y}-\left\llcorner\mu^{*}\left(K_{X}+\Delta\right)+c A\right\lrcorner\right) .
$$

The expression on the right does not make sense a priori, since it depends on the divisor chosen to represent the canonical class (rounding does not commute with numerical equivalence). But this definition can be made rigorous using discrepancies as explained in [Laz, Chapter 9.3.F]. In the case of toric varieties there is a canonical torus invariant representative $K_{X}$ of the canonical class. Namely, if $D_{1}, \ldots, D_{s}$ are the torus invariant prime Weil divisors on $X$ then $K_{X}=-\left(D_{1}+\ldots+D_{s}\right)$. Using this choice for $K_{X}$ and $K_{Y}$, one easily verifies that $K_{Y}-\mu^{*}\left(K_{X}+\Delta\right.$ ) is equal (not

Date: October 29, 2003

2000 Mathematics Subject Classification. 14J17,13A35.

Key words and phrases. multiplier ideal, toric variety, test ideal, tight closure.

The author is grateful to Nobuo Hara for interesting discussions and thanks the referee for a careful reading and thoughtful comments. 
just numerically equivalent) to $E-\widetilde{\Delta}$, where $E$ is $\mu$-exceptional and $\widetilde{\Delta}$ denotes the strict transform of $\Delta$. This validates our setup.

Now let $(X, \Delta)$ be a pair such that $X$ is an (affine) toric variety (say $X=\operatorname{Spec} R$ for some normal semigroup ring $\left.A \subseteq k\left[x_{1}^{ \pm 1}, \ldots, x_{n}^{ \pm 1}\right]\right)$ and $\Delta$ is a torus invariant $\mathbb{Q}$-divisor. Since $K_{X}+\Delta$ is $\mathbb{Q}$-Cartier and torus invariant, there is a monomial $x^{u}$ such that $\operatorname{div} x^{u}=r\left(K_{X}+\Delta\right)$ for some integer $r$. With $w=u / r$, one obtains the following result:

Theorem 1. Let $\mathfrak{a}$ be a monomial ideal on $X$. Then

$$
\left.\mathcal{J}\left((X, \Delta), \mathfrak{a}^{c}\right)=\left\langle x^{v} \in R\right| v+w \in \text { interior of } c \operatorname{Newt}(\mathfrak{a})\right\rangle
$$

for all $c>0$.

If $X=\mathbb{A}^{n}$ and $\Delta=\emptyset$ this is just Howald's forlmula. If $X$ is $\mathbb{Q}$-Gorenstein and $\Delta=\emptyset$ this recovers a generalization by Hara and Yoshida $[\mathrm{HY}$ obtained with positive characteristic methods; also Howard Thompson obtained this case independently.

1.2. Multiplier module. A variant of the multiplier ideal on a singular variety is the multiplier module. It has the advantage to be defined on any normal variety, not relying of a $\mathbb{Q}$-Gorenstein assumption, see, for example, HS02. The multiplier module is no longer an ideal but a submodule of the canonical module $\omega_{X}$.

Definition 2. Let $X$ be a normal variety and let $\mathfrak{a}$ be a sheaf of ideals on $X$. Let $\mu: Y \rightarrow X$ be a $\log$ resolution of $\mathfrak{a}$ such that $\mathfrak{a} \cdot \mathcal{O}_{Y}=\mathcal{O}_{Y}(-A)$. Then the multiplier module is defined as

$$
\mathcal{J}_{\omega}\left(\mathfrak{a}^{c}\right)=\mu_{*} \mathcal{O}_{Y}\left(K_{Y}-\llcorner c A\lrcorner\right) \subseteq \omega_{X}
$$

for all $c>0$.

This is independent of the chosen $\log$ resolution of $\mathfrak{a}$. For an affine toric variety $X$ there is a canonical embedding of $\omega_{X} \subseteq \mathcal{O}_{X}$, which realizes $\omega_{X}$ as a monomial ideal. In this canonical description of $\omega_{X}$ the formula computing the multiplier module takes a stunningly easy form.

Theorem 2. Let $X$ be an affine toric variety and $\mathfrak{a}$ a monomial ideal. Then

$$
\left.\mathcal{J}_{\omega}\left(\mathfrak{a}^{c}\right)=\left\langle x^{v}\right| v \in \text { interior of } c \operatorname{Newt}(\mathfrak{a})\right\rangle \subseteq \omega_{X}
$$

for all $c>0$.

Clearly, as multiplier ideals are local in nature one can derive similar statements for the multiplier ideal or module of an arbitrary normal toric variety by computing on an affine toric cover using the above formulas. In general the notion of multiplier module and ideal require the characteristic to be zero since their construction employs resolution of singularities. Since for toric varieties log resolutions are available also in positive characteristic, the preceding results are valid, independent of the characteristic.

1.3. Positive characteristic: Test ideals. In positive characteristic, Hara and Yoshida introduced a related notion of the test ideal $\tau\left(\mathfrak{a}^{c}\right)$ which does not require a $\mathbb{Q}$-Gorenstein assumption. They also show that in the $\mathbb{Q}$-Gorenstein case the multiplier ideal $\mathcal{J}\left(\mathfrak{a}^{c}\right)$ generically yields their test ideal $\tau\left(\mathfrak{a}^{c}\right)$ under reduction to positive characteristic. As a final result I give a formula (see Theorem 3 ) for the test 
ideal $\tau\left(\mathfrak{a}^{c}\right)$ for an affine toric variety defined over a field of positive characteristic. This formula specializes to the formula above in the $\mathbb{Q}$-Gorenstein case. We relegate the statement (Theorem 31) and proof to the last section since we first need to recall some basics from toric geometry.

\section{TORIC SETUP}

Following Fulton Ful93, I fix a dual pair of lattices $N=M^{\vee} \cong \mathbb{Z}^{n}$. Let $\sigma \subseteq N_{\mathbb{R}}=N \otimes_{\mathbb{Z}} \mathbb{R}$ be a strongly convex rational polyhedral cone given by $\sigma=$ $\left\{r_{1} u_{1}+\ldots+r_{t} u_{t} \mid r_{i} \in \mathbb{R}_{+}\right\}$for some $u_{1}, \ldots, u_{t} \in N$. The dual cone $\sigma^{\vee}$ is a (rational convex polyhedral) cone in $M_{\mathbb{R}}$ defined by $\sigma^{\vee}=\left\{m \in M_{\mathbb{R}} \mid(m, v) \geq 0, \forall v \in \sigma\right\}$ where $(\cdot, \cdot)$ denotes the pairing of the dual lattices $M$ and $N$. The lattice points in $\sigma^{\vee}$ give rise to a sub-semigroup of Laurent polynomials $k\left[x_{1}^{ \pm 1}, \ldots, x_{n}^{ \pm 1}\right]$ generated by those monomials $x^{m}=x_{1}^{m_{1}} \cdot \ldots \cdot x_{n}^{m_{n}}$ such that $m \in \sigma^{\vee}$. Identifying a monomial with its exponent this yields the affine semigroup ring

$$
R_{\sigma}=k\left[\sigma^{\vee} \cap M\right] .
$$

The affine toric variety is $X_{\sigma}=\operatorname{Spec} R_{\sigma}$. Since $R_{\sigma}$ is contained in the ring of Laurent polynomials, $X_{\sigma}$ contains the torus $\left(k^{*}\right)^{n}=T^{n}=\operatorname{Spec} k\left[x_{1}^{ \pm 1}, \ldots, x_{n}^{ \pm 1}\right]$ as a dense subset. The action of the torus on itself extends naturally to an action on the whole of $X_{\sigma}$.

An ideal $\mathfrak{a}$ of a toric ring $R_{\sigma}$ is invariant under the action of the torus if and only if it is generated by monomials, that is, if it is a monomial ideal. To such ideal $\mathfrak{a}$ one associates its Newton polyhedron $\operatorname{Newt}(\mathfrak{a}) \subseteq M_{\mathbb{R}}$, defined as the convex hull of $\mathfrak{a}$, or more precisely the convex hull of the set of exponents $m$ of the monomials $x^{m}$ in $\mathfrak{a}$.

2.1. Divisors. The prime (Weil) divisors which are fixed by this torus action have an easy description, they correspond to the edges (or rays) of the cone $\sigma$. Let $v_{1}, \ldots, v_{s}$ be the first lattice points on the edges of $\sigma$. Their orthogonals $v_{i}^{\perp} \cap \sigma^{\vee} \subseteq$ $\sigma^{\vee}$ are the facets of $\sigma^{\vee}$. They define codimension one subvarieties $D_{i}$ of $X_{\sigma}$. Any torus invariant divisor can be written as a sum of the $D_{i}$ and we denote the lattice of torus invariant divisors by $L=L^{X_{\sigma}}=\oplus \mathbb{Z} D_{i}$. A torus invariant Cartier divisor can be written as $\operatorname{div} x^{m}$ for a Laurent monomial $x^{m}$. The fact that $\operatorname{ord}_{D_{i}} x^{m}=\left(m, v_{i}\right)$ shows therefore that a divisor $D=\sum d_{i} D_{i}$ is $\mathbb{Q}$-Cartier if and only if there is $w \in M_{\mathbb{Q}}$ such that $\left(w, v_{i}\right)=d_{i}$ for all $i$.

There is a canonical choice of a divisor $K_{X_{\sigma}}$ to represent the canonical class. Namely the torus invariant divisor which is just the negative of the sum of the prime divisors $D_{i}$, that is $K_{X_{\sigma}}=-\sum D_{i}$. With this canonical divisor, the canonical module $\omega_{X_{\sigma}}$ is the ideal of $R_{\sigma}$ consisting precisely of the monomials $x^{m}$ such that $m$ is in the interior of $\sigma^{\vee}$. In other words, $x^{m} \in \omega_{X_{\sigma}}$ if and only if $\operatorname{ord}_{D_{i}} x^{m}=$ $\left(m, v_{i}\right)>0$ for all $i$.

A general toric variety is made up from affine pieces via the datum of a fan $\Sigma$. A fan is a collection of compatible cones (any two cones in $\Sigma$ meet in a common face ...). The considerations made above are also valid for this non affine setting, refer to [Ful93] or Dan78 for justification of all of the above (and all that follows in this section). 
2.2. Resolutions. A toric variety $X$ can be desingularized via an easy combinatorial procedure involving subdividing the fan $\Sigma$ to arrive at a fan $\Sigma^{\prime}$ all of whose maximal cones are spanned by a basis of $N$. We only need to know that this yields a torus equivariant desingularization $\mu: Y \rightarrow X$ where $Y$ is the toric variety defined by $\Sigma^{\prime}$. Similarly, log resolutions of an ideal are also obtained torically. Since the monomial ideals are precisely the torus invariant ideals, it follows that the multiplier ideals of monomial ideals will also be monomial ideals.

Recall that toric varieties have at worst rational singularities, therefore, $\mu_{*} \omega_{Y}=$ $\omega_{X}$. This has the following immediate consequence which will be used later.

Lemma 1. Let $\mu: Y \rightarrow X$ be a toric resolution of singularities. If $x^{m} \in \omega_{X}$ then $\operatorname{ord}_{D_{i}} \mu^{*} x^{m}>0$ for $D_{i}$ any torus invariant prime Weil divisor on $Y$. Consequently, $\operatorname{Supp}\left(\mu^{*} \operatorname{div} x^{m}\right)$ and $K_{Y}$ have the same support.

Proof. The condition that $x^{m}$ is a section of $\omega_{X}$ implies by $\mu_{*} \omega_{Y}=\omega_{X}$ that $x^{m}$ (viewed on $Y$ ) is a section of $\omega_{Y}$. But this just means $\operatorname{ord}_{D_{i}} \mu^{*} x^{m}>0$ for all $i$ as claimed.

Another simple observation is the following lemma.

Lemma 2. Let $\mu: Y \rightarrow X$ be a toric log resolution of the monomial ideal $\mathfrak{a}$ such that $\mathfrak{a} \cdot \mathcal{O}_{Y}=\mathcal{O}_{Y}(-A)$. For a monomial $x^{m}$ one has

$$
c m \in c^{\prime} \operatorname{Newt}(\mathfrak{a}) \Longleftrightarrow c \mu^{*} \operatorname{div} x^{m} \geq c^{\prime} A .
$$

for all rational (real) numbers $c$ and $c^{\prime}$.

Proof. It is well known Ful93 that $m \in \operatorname{Newt}(\mathfrak{a})$ if and only if $x^{m} \in \overline{\mathfrak{a}}$, the integral closure of $\mathfrak{a}$. Since $\overline{\mathfrak{a}}=\mu_{*} \mathcal{O}_{Y}(-A)$, this is equivalent to $\mu^{*} \operatorname{div} x^{v} \geq A$. This was the case $c=c^{\prime}=1$ and the rest follows easily by expressing $c$ and $c^{\prime}$ as integer fractions and clearing denominators.

With these preparations we proceed to the proofs of Theorem 1 and Theorem 2

\section{Proofs of Theorem $1 \& 2$}

Both proofs are essentially the same argument. I first give the proof for Theorem 2 and then indicate the small changes needed in the proof of Theorem 1

Proof of Theorem [2 Fix a toric $\log$ resolution $\mu: Y \rightarrow X_{\sigma}$ of $\mathfrak{a}$ such that $\mathfrak{a} \cdot \mathcal{O}_{Y}=$ $\mathcal{O}_{Y}(-A)$. For $m$ to be in the interior of the Newton polyhedron $c \operatorname{Newt}(\mathfrak{a})$ is the same as

$$
m-\varepsilon m^{\prime} \in c \operatorname{Newt}(\mathfrak{a})
$$

for all (some) $m^{\prime} \in M$ in the interior of $\sigma^{\vee}$ and all small enough (rational) $\varepsilon$. This is because if $z \in \operatorname{Newt}(\mathfrak{a})$, then $z+\sigma^{\vee} \subseteq \operatorname{Newt}(a)$. Applying Lemma 2 we get that this is equivalent to

$$
\mu^{*} \operatorname{div} x^{m}-\varepsilon \mu^{*} \operatorname{div} x^{m^{\prime}} \geq c A,
$$

which is, since $\mu^{*} \operatorname{div} x^{m}$ is integral, equivalent to

$$
\mu^{*} \operatorname{div} x^{m} \geq\left\ulcorner\varepsilon \mu^{*} \operatorname{div} x^{m^{\prime}}+c A\right\urcorner .
$$

By Lemma 1 (using that $m^{\prime}$ is in the interior of $\sigma^{\vee}$ if and only if $x^{m^{\prime}} \in \omega_{X}$ ), the $\mathbb{Q}$-divisor $\varepsilon \mu^{*} \operatorname{div} x^{m^{\prime}}$ is effective with support equal to $-K_{Y}$, that is the prime 
divisors in $\mu^{*} \operatorname{div} x^{m^{\prime}}$ are precisely all the torus invariant divisors. Then an exercise in rounding shows that the last inequality is equivalent to

$$
\mu^{*} \operatorname{div} x^{v} \geq K_{Y}+\llcorner c A\lrcorner
$$

as claimed.

Proof of Theorem [1 Let $\mu: Y \rightarrow X$ be a toric log resolution as in the last proof. The condition that $m+w$ is in the interior of the Newton polyhedron Newt(a) is equivalent to $m+u / r-\varepsilon m^{\prime} \in \operatorname{Newt}(\mathfrak{a})$ for $m^{\prime}$ in the interior of $\sigma$ and small enough rational $\varepsilon>0$. Again, by Lemma 2 this is equivalent to

$$
\mu^{*} \operatorname{div} x^{m}+\frac{1}{r} \mu^{*} \operatorname{div} x^{u}-\varepsilon \mu^{*} \operatorname{div} x^{m^{\prime}} \geq c A .
$$

Using that $\operatorname{div} x^{u}=r\left(K_{X}+\Delta\right)$ and proceeding analogous to the last proof this is equivalent to

$$
\mu^{*} \operatorname{div} x^{m} \geq K_{Y}-\left\llcorner\mu^{*}\left(K_{X}+\Delta\right)+c A\right\lrcorner
$$

which says, by definition, nothing but that $x^{m} \in \mathcal{J}\left((X, \Delta) ; \mathfrak{a}^{c}\right)$.

\section{Formula For the test ideal And SPECUlations}

In positive characteristic, Hara and Yoshida $\mathrm{HY}$ introduced a notion of test ideal which, under the process of reduction to positive characteristic corresponds to the multiplier ideal. This notion grew out of tight closure theory Hun96, where test ideals play an important role. Their definition does not rely on a $\mathbb{Q}$-Gorenstein assumption, which can be attributed to the fact that their construction does not use resolutions of singularities. With their test ideal $\tau\left(\mathfrak{a}^{c}\right)$ replacing the multiplier ideal one obtains a result for the test ideal of a monomial ideal on an arbitrary affine normal toric variety over a field of positive characteristic.

Theorem 3. Let $X_{\sigma}=\operatorname{Spec} R$ be a toric variety over a field of positive characteristic and $\mathfrak{a}$ a monomial ideal. Then a monomial $x^{m} \in R$ is in $\tau\left(\mathfrak{a}^{c}\right)$ if and only if there exists $w \in M_{\mathbb{R}}$ with $\left(w, v_{i}\right) \leq 1$ for all $i$, such that

$$
m+w \in \text { interior of } c \operatorname{Newt}(\mathfrak{a}) \text {. }
$$

If $R$ is $\mathbb{Q}$-Gorenstein, then there is a $w_{0}$ with $\left(w_{0}, v_{i}\right)=1$ for all $i$. Therefore $x^{m} \in \tau\left(\mathfrak{a}^{c}\right)$ if and only if $m+w_{0} \in$ interior of $c \operatorname{Newt}(\mathfrak{a})$.

The definition of the test ideal associated to an ideal $\mathfrak{a} \subseteq R$ and a rational parameter $c$ is

$$
\tau\left(\mathfrak{a}^{c}\right) \stackrel{\text { def }}{=}\left\{h \in R \mid h I^{* \mathfrak{a}^{c}} \subseteq I \text { for all ideals } I \text { of } R\right\} .
$$

The $\mathfrak{a}^{c}$-tight closure appearing is defined similarly as the usual tight closure: $x \in$ $I^{* a^{c}}$ if there is an $h \in R^{\circ}$ such that for all $q=p^{e}$ one has $h x^{q} \mathfrak{a}^{\ulcorner c q\urcorner} \subseteq I^{[q]}$. I will not elaborate on the properties of $\mathfrak{a}^{c}$-tight closure and the resulting test ideals (see $[\mathrm{HY}]$ ) but instead mention the one result needed to prove Theorem 3

Lemma 3. Let $(R, \mathfrak{m})$ be a graded ring and $\mathfrak{a}$ a homogeneous ideal. Then

$$
\tau\left(\mathfrak{a}^{c}\right)=\operatorname{Ann}_{R}\left(0_{E_{R / \mathfrak{m}}}^{* \mathfrak{a}^{c}}\right),
$$

where $E_{R / \mathfrak{m}}$ is the injective hull of the residue field of $R$. 
Proof. First observe that Theorem 3.3 of [LS99] (saying that in the graded case and for an Artinian module the finitistic tight closure (which is a variant of tight closure) is equal to the tight closure) can easily be adapted to the case of $\mathfrak{a}^{c}$-tight closure, provided that $\mathfrak{a}$ is also graded. Since semigroup rings are in particular $\mathbb{N}$-graded and so are all our modules and ideals involved, this implies that

$$
0_{E}^{* \mathfrak{a}^{c}}=\bigcup_{\substack{\text { finitely } \\ \text { generated } \\ V \subseteq E}} 0_{V}^{* \mathfrak{a}^{c}} .
$$

As by $[\mathrm{HY}]$, Proposition 1.9, the test ideal $\tau\left(\mathfrak{a}^{c}\right)$ is the annihilator of the right hand side the result follows.

With this observation we can modify the proof of Theorem 4.8 of $\underline{\mathrm{HY}}$ to apply also to the non $\mathbb{Q}$-Gorenstein situation.

Proof of Theorem 3 . We recall the description of the injective hull $E$ of the residue field in the case of a toric ring. It is the (graded) Matlis dual of $R$. Since $R$ consists of the monomials $x^{m}$ such that $\left(m, v_{i}\right) \geq 0$ for all $i$, its dual $E$ consist of the monomials $x^{m}$ such that $\left(m, v_{i}\right) \leq 0$. By Wat91, Theorem $2.5, F^{* e}(E)$ is dual to $\omega_{R}^{\left(p^{e}-1\right)}$ where the latter consists of all $m \in M$ such that $\left(m, v_{i}\right) \geq 1-p^{e}$ for all $i$. Therefore,

$$
\left.F^{* e}(E)=\left\langle x^{m}\right|\left(m, v_{i}\right) \leq p^{e}-1 \text { for all } i\right\rangle .
$$

An easy consequence of Lemma 3 is that $x^{m} \in \tau\left(\mathfrak{a}^{c}\right) \Longleftrightarrow x^{-m} \notin 0_{E}^{* \mathfrak{a}^{c}}$. Clearly, since $x^{m} \cdot x^{-m}=1$ is nonzero in $E$ the implication from left to right is clear. Conversely, if $x^{m} \notin \tau\left(\mathfrak{a}^{c}\right)=\operatorname{Ann}_{R} 0_{E_{R / m}^{*} \mathfrak{a}^{c}}$ there is $x^{m^{\prime}} \in 0_{E_{R / m}^{* \mathfrak{a}^{c}}}$ such that $x^{m+m^{\prime}} \neq 0$ in $E_{R / m}$. Therefore, $x^{-\left(m+m^{\prime}\right)} \neq 0$ in $R$ and consequently, $x^{-m}=x^{-\left(m+m^{\prime}\right)} x^{m^{\prime}}$ is in $0_{E_{R / m}}^{* \mathfrak{a}^{c}}$. Now the following chain of equivalences shows the result.

$$
\begin{aligned}
x^{m} \in \tau\left(\mathfrak{a}^{c}\right) & \Longleftrightarrow x^{-m} \notin 0_{E}^{* \mathfrak{a}^{c}} \Longleftrightarrow \exists q: 1 \cdot x^{-q m} \mathfrak{a}^{\ulcorner c q\urcorner} \neq 0 \text { in } F^{* e}(E) \\
& \Longleftrightarrow \exists q:(-q m+\ulcorner c q\urcorner \operatorname{Newt}(\mathfrak{a})) \cap\left\{m^{\prime} \mid \forall i\left(m^{\prime}, v_{i}\right) \leq q-1\right\} \cap M \neq \emptyset \\
& \Longleftrightarrow \exists q \exists w: q m+q w \in\ulcorner c q\urcorner \operatorname{Newt}(\mathfrak{a}) \cap M \text { and } \forall i\left(q w, v_{i}\right) \leq(q-1) \\
& \Longleftrightarrow \exists q, w: m+w \in \frac{\ulcorner c q\urcorner}{q} \operatorname{Newt}(\mathfrak{a}) \cap \frac{1}{q} M \text { and } \forall i\left(w, v_{i}\right) \leq 1-\frac{1}{q} \\
& \Longleftrightarrow \exists w: \forall i\left(w, v_{i}\right) \leq 1 \text { and } m+w \in \operatorname{Int}\left(\operatorname{Newt}\left(\mathfrak{a}^{c}\right)\right)
\end{aligned}
$$

The second equivalence is just the definition of $\mathfrak{a}^{c}$ tight closure for the zero submodule using that a toric ring is strongly $F$-regular, and thus 1 can be used as a test element [HY] Theorem 1.7]. The only other implication that needs explanation is the reverse of the last equivalence. For this let $w$ be as in the last line. Since $m+w$ is in the interior we can perturb $w$ slightly such that for large enough $q$ we have $q w \in M,\left(w, v_{i}\right) \leq 1-\frac{1}{q}$ and $m+w \in\left(c+\frac{1}{q}\right) \operatorname{Newt}(\mathfrak{a}) \subseteq \frac{\ulcorner c q\urcorner}{q} \operatorname{Newt}(\mathfrak{a})$ as required.

Finally, $R$ is $\mathbb{Q}$-Gorenstein if and only if there is $w_{0}$ such that for all $v_{i}$ one has $\left(w_{0}, v_{i}\right)=1$. Then, clearly, the last condition is equivalent to

$$
m+w_{0} \in \operatorname{Int}\left(\operatorname{Newt}\left(\mathfrak{a}^{c}\right)\right)
$$

as claimed. 
Note that Theorems 1 and 3 also give a direct argument in the toric case for the fact that $\tau\left(\mathfrak{a}^{c}\right)=\mathcal{J}\left(\mathfrak{a}^{c}\right)$ if $X$ is $\mathbb{Q}$-Gorenstein. More generally one can make the following observation, which was pointed out by N. Hara:

Corollary. Let $X$ be an affine toric variety over a field of positive characteristic and $\mathfrak{a}$ a monomial ideal. Then

$$
\tau\left(X, \mathfrak{a}^{c}\right)=\sum \mathcal{J}\left((X, \Delta), \mathfrak{a}^{c}\right)
$$

where the sum ranges over all effective torus invariant $\mathbb{Q}$-divisors $\Delta$ such that $K_{X}+$ $\Delta$ is $\mathbb{Q}$-Cartier.

Proof. If $w \in M_{\mathbb{Q}}$ and $\Delta$ are related by the formula $\Delta=\sum\left(1-\left(w, v_{i}\right)\right) D_{i}$ we have that $K_{X}+\Delta=-\operatorname{div} x^{w}$ is $\mathbb{Q}$-Cartier and that $\Delta$ is effective if and only if $\left(w, v_{i}\right) \leq 1$ for all $i$. By Theorem [3, $x^{m} \in \tau\left(X, \mathfrak{a}^{c}\right)$ if and only if there is $w \in M_{\mathbb{Q}}$ such that $\left(w, v_{i}\right) \leq 1$ and $m+w \in \operatorname{Int}\left(\operatorname{Newt}\left(\mathfrak{a}^{c}\right)\right)$. With $\Delta$ as above this is equivalent to $x^{m} \in \mathcal{J}\left((X, \Delta), \mathfrak{a}^{c}\right)$ by Theorem 1

Furthermore, it should be straightforward to define $\tau\left((X, \Delta), \mathfrak{a}^{c}\right)$ in positive characteristic analogous to $\mathcal{J}\left((X, \Delta), \mathfrak{a}^{c}\right)$ in characteristic zero. Then a test ideal version of Theorem 1 should hold. This would lead to the statement, in analogy with the Corollary, that

$$
\tau\left(X, \mathfrak{a}^{c}\right)=\sum_{\substack{\Delta \text { effective, } \\ K_{X}+\Delta \text { is } \mathbb{Q} \text {-Cartier. }}} \tau\left((X, \Delta), \mathfrak{a}^{c}\right)
$$

for any toric variety $X$. This naturally leads to the following question: What is the class of varieties such that equation (11) holds?

On the other hand, in an effort to enlarge the definition of the multiplier ideal beyond the $\mathbb{Q}$-Gorenstein case, formula (1) for the test ideals (which does not depend on a $\mathbb{Q}$-Gorenstein assumption) can serve as a guideline. One is lead to speculate whether the following definition leads to a useful generalization of the multiplier ideal, say if $X$ is over a field of characteristic zero we set

$$
\mathcal{J}\left(X, \mathfrak{a}^{c}\right) \stackrel{\text { def }}{=} \sum_{\substack{\Delta \text { effective, } \\ K_{X}+\Delta \text { is } \mathbb{Q} \text {-Cartier. }}} \mathcal{J}\left((X, \Delta), \mathfrak{a}^{c}\right)
$$

Clearly, in the $\mathbb{Q}$-Gorenstein case this recovers the definition of $\mathcal{J}\left((X, \emptyset), \mathfrak{a}^{c}\right)$ above. This definition would be justified, for example, if $\mathcal{J}\left(X, \mathfrak{a}^{c}\right)$ satisfies a Nadel type vanishing theorem. At the same time it is clear that one still needs to control the singularities of $X$, at least one has to require that there exists an effective $\mathbb{Q}$-divisor $\Delta$ such that $K_{X}+\Delta$ is $\mathbb{Q}$-Cartier in order for the definition to make sense.

\section{REFERENCES}

[Dan78] V. I. Danilov, The geometry of toric varieties, Uspekhi Mat. Nauk 33 (1978), no. 2(200), 85-134, 247.

[Ful93] William Fulton, Introduction to toric varieties, Annals of Mathematics Studies, vol. 131, Princeton University Press, Princeton, NJ, 1993, The William H. Roever Lectures in Geometry.

[How01] J. A. Howald, Multiplier ideals of monomial ideals, Trans. Amer. Math. Soc. 353 (2001), no. 7, 2665-2671 (electronic).

[HS02] Eero Hyry and Karen Smith, On a non-vanishing conjecture of Kawamata and the core of an ideal, submitted, 2002. 
[Hun96] Craig Huneke, Tight closure and its applications, CBMS Regional Conference Series in Mathematics, vol. 88, Published for the Conference Board of the Mathematical Sciences, Washington, DC, 1996, With an appendix by Melvin Hochster.

[HY] Nobuo Hara and Ken-ichi Yoshida, A generalization of tight closure and multiplier ideals, arXiv:math.AC/0211008.

[Laz] Robert Lazarsfeld, Positivity in algebraic geometry, Monograph in preparation.

[LS99] Gennady Lyubeznik and Karen E. Smith, Strong and weak F-regularity are equivalent for graded rings., Am. J. Math. 121 (1999), no. 6, 1279-1290.

[Wat91] Kei-ichi Watanabe, F-regular and F-pure normal graded rings., Journal of Pure and Applied Algebra 71 (1991), 341-350 (English).

Universität Essen, FB6 Mathematik, 45117 Essen, Germany

E-mail address: manuel.blickle@uni-essen.de

$U R L$ : www.mabli.org 